\title{
Investigation of the Magnetic Response of a Nanocrystalline High-Frequency Magnetic-Link with Multi-input Excitations
}

\author{
Md. Rabiul Islam, Senior Member, IEEE, Md. Ashib Rahman, Member, IEEE, Pejush Chandra Sarker, \\ Kashem M. Muttaqi, Senior Member, IEEE, and Danny Sutanto, Senior Member, IEEE
}

\begin{abstract}
With the technological advancement of magnetic materials and semiconductor devices, the use of high-frequency magnetic-link made of high-saturation induction and low specific core loss nanocrystalline alloy has been gaining popularity to replace the common dc-link or ac-link specially in integrating multiple renewable sources to the grid. The magnetic integration of multiple renewable sources through a common magnetic-link has a number of advantages including galvanic isolation compared with the common dc or ac-link. However, the magnetic response of the common magnetic-link under multi-input excitations highly affects the energy conversion efficiency. To the best of the authors' knowledge, the magnetic response of an advanced magnetic material-based magnetic-link under multiinput excitations has not been well investigated in the literature. In this paper, a systematic investigation is carried out to interconnect multiple sources through a common magnetic-link. The magnetic response and the procedure used in the investigation are also presented and discussed in this paper.
\end{abstract}

Index Terms-high-frequency magnetic-link, multi-input excitations, magnetism, magnetic integration, renewable sources.

\section{INTRODUCTION}

$\mathrm{R}$ APIDLY diminishing reserve of fossil fuels and increasing of environmental degradation have accelerated the development of new technologies to optimize the use of natural resources and to use nonconventional sources to serve increasing load demands [1], [2]. In this regard, industry and academia have been developing emerging technologies such as high-temperature superconducting (HTS) generators for large wind farms [3], [4], HTS cables for high-voltage transmission lines [5], [6], HTS power devices for sub-stations [7], HTS machines for transportations [8], [9], and a new variety of power converters for smart micro-grid [10], electric vehicles [11], and for grid connected renewable generation systems [12]. The high-frequency magnetic-link has become

Manuscript submission for review, September 30, 2018.

M. R. Islam, M. A. Rahman, K. M. Muttaqi, and D. Sutanto are with the School of Electrical, Computer and Telecommunications Engineering, Faculty of Engineering and Information Sciences, University of Wollongong, NSW 2522, Australia. e-mail: mrislam@uow.edu.au, mar997@uowmail.edu.au, kashem@uow.edu.au, soetanto@uow.edu.au.

P. C. Sarker is with School of Electrical and Data Engineering, University of Technology Sydney, Broadway NSW 2007, Australia. E-mails: PejushChandra.Sarker@student.uts.edu.au.

Color versions of one or more of the figures in this paper are available online at http://ieeexplore.ieee.org.

Digital Object Identifier will be inserted here upon acceptance. one of the promising technologies in designing new power converters due to its unparalleled features, such as galvanic isolation and voltage balancing capabilities. The dual active bridge (DAB) dc-dc converter is one of the basic and simplest example where high-frequency magnetic-link can be applied [13]. A DAB converter uses a magnetic-link with only two windings that is connected to either a half or a full-bridge inverter. The direction of the power flow can be controlled by shifting the phases of the inverters. The dual port converter topology has already been extended to a multi-port converter with a multi-winding magnetic-link for use in multi-mode operations [14], [15]. The literature mainly focused on the design of the power converter circuits and the switching techniques to control the powers of the multi-port converter outputs.

In recent years, the use of medium-voltage multilevel converters have been found to be one of the promising technology specially for use in the step-up transformer-less direct grid integration of renewable sources, industrial motor drives and for solid-state transformer (SST) applications [16][18]. Most of the multilevel converter topologies require multiple isolated and balanced dc supplies and (or) needs to balance the capacitor voltages. It is already proven that multiwinding high-frequency magnetic-link can inherently overcome these issues and has the capability to generate multiple isolated and balanced dc supplies from single or multiple sources [19]. Although, the design optimization of such a high-frequency magnetic-link with advanced magnetic materials has been presented in [20], as far as the authors are aware, no report has been reported in the literature that has investigated the magnetic response of the magnetic link under multi-input excitations.

Recently, a common magnetic-bus instead of common dcor ac-bus has been proposed to interconnect multiple power sources such as solar photovoltaic modules, wind turbines, fuel cells, and energy storage unites magnetically, which eliminates the direct electrical connections between different sources [21]. The multi-winding high-frequency magnetic-bus uses power converters to interconnect and control the power flows among various input sources with the grid and the load. The magnetic integration of multiple renewable sources through a common magnetic link has a number of advantages including galvanic isolation when compared with the use of a common dc-link.

Although recent development of magnetic materials such as 
amorphous and nanocrystalline alloys enable the design of a compact, lightweight and efficient high-frequency magnetic link/bus, but the overall power conversion efficiency highly depends on the magnetic response of the multi-input magnetic-link. Therefore, a systematic analysis of magnetic response of a multi-input magnetic-link made of nanocrystalline alloy is necessary to design the switching and control circuit of the high-frequency magnetic-link based power converters. In this paper, a systematic investigation is carried out to interconnect multiple sources through a common magnetic-link. This paper presents and discusses the detailed procedure of investigation of magnetic response under multiple excitations. It is expected that the paper will be a good reference for future designers of high-frequency magnetic-link based power converters.

\section{Theoretical Modeling of a Multi-INPUt Magnetic-} LINK

In this section, a three-port high-frequency magnetic link, as shown in Fig. 1(a) is considered for theoretical analysis. A separate winding is implied for each port of the magnetic-link.

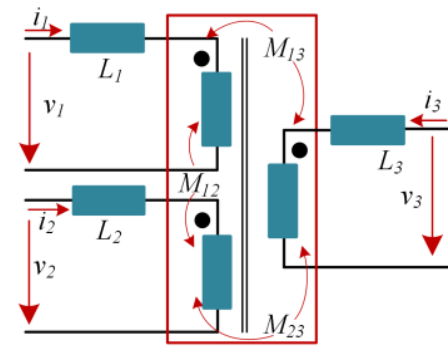

(a)

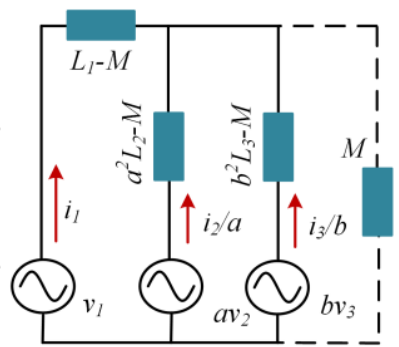

(b)
Fig. 1. (a) Three-port magnetically isolated power supply system and (b) simplified circuit with decoupled mutual flux linkage (magnetization inductance $L_{m}$ is omitted).

The simplified dynamic equation of the three-port magnetic link can be represented as

$$
\left[\begin{array}{c}
v_{1}(t) \\
a v_{2}(t) \\
b v_{3}(t)
\end{array}\right]=\left[\begin{array}{ccc}
L_{1} & M & M \\
M & a^{2} L_{2} & M \\
M & M & b^{2} L_{3}
\end{array}\right]\left[\begin{array}{c}
\frac{d i_{1}(t)}{d t} \\
\frac{d}{d t}\left(\frac{i_{2}}{a}\right) \\
\frac{d}{d t}\left(\frac{i_{3}}{b}\right)
\end{array}\right]
$$

where $v_{1}, v_{2}$, and $v_{3}$ are the input voltages of winding 1,2 , and 3 , respectively. The corresponding currents of the three windings are defined by $i_{1}, i_{2}$, and $i_{3} . L_{1}, L_{2}$, and $L_{3}$ are the leakage inductances of the three windings. The mutual inductances are defined by the parameter $M$, with the assumption that $a M_{12}=b M_{13}=a b M_{23}=M$, where subscripts denote the winding numbers. The simplified model of the three-port magnetic link is shown in Fig. 1(b).

\section{INVESTIGATION IN ANSYS ENVIRONMENT AND DISCUSSIONS}

To carry out a systematic investigation, a three windings high-frequency magnetic link is modelled in ANSYS environment, as shown in Fig. 2. Winding 1 and winding 2 are excited by two sources, and the third winding is considered as the output winding. For the same voltage amplitude, phase and frequency, the resultant magneto-motive force is produced equally by the two sources and they also share the load equally. However, the power capacities of sources are not generally equal. Therefore, the load shared by the sources is not the same. To change the load sharing, at least one of the electrical characteristics such as the voltage amplitude, the phase or the frequency can be changed.



Fig. 2. Multi-winding magnetic-link in ANSYS environment.

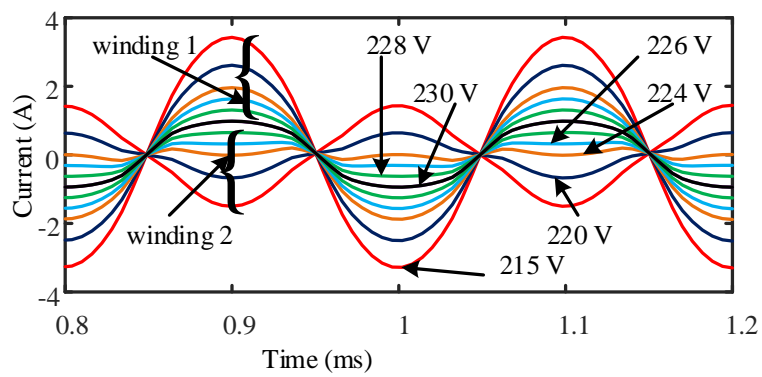

Fig. 3. The effect of voltage amplitude changes on the winding currents.

The effect of the voltage amplitude change on the winding currents under no-load condition is shown in Fig. 3. For the analysis, the voltage of the winding 1 is kept constant and the voltage of the winding 2 is varied. Winding 1 is excited by a fixed $230 \mathrm{~V}$ and the excitation voltage of winding 2 is decreased gradually. When the two voltages are the same, the no-load currents in both windings are the same in both amplitude and phase. When the voltage on winding 2 decreases to $228 \mathrm{~V}$, the winding 2 no-load current decreases. On the other hand, the no-load current increases in winding 1. As the voltage amplitude decreases, the winding 2 current decreases further and the winding 1 current increases. This situation can be directly observed from the simplified diagram of the three-port magnetic link shown in Fig. 1(b) based on (1). For the unity turns ratio $(a=b=c=1)$ and if the winding 3 is left open, then from (1), with the variation of the voltage of $v_{1}$ and $v_{2}$, the rate of change of the exciting currents and hence the exciting current, $i_{1}$, and $i_{2}$, will vary accordingly. If the winding 1 voltage is higher than the voltage in winding 2 , winding 1 will carry higher no-load current than that of winding 2 , and vice versa. At a certain voltage, the phase of winding 2 current becomes the opposite of that of winding 1 current. At this point, the winding 1 produces only magneto 
motive force. As the voltage is further decreased on winding 2 , the no load currents increase in both windings, and under this condition, winding 2 works as a load and receives power from the excitation of winding 1 .

To observe the effect of the phase change of the voltage, different phase differences between two sources are applied on windings 1 and 2. The simulation results in Fig. 4 show that, the currents in both windings also increase, as an extra current also flows through the windings due to the phase changes. The flux distribution becomes non-uniform along the core. For the $5^{\circ}$ and $0^{\circ}$ phase differences between two excited voltages, the flux densities at time $t=0.00085 \mathrm{~s}$, are shown in Fig. 5 and Fig. 6, respectively.

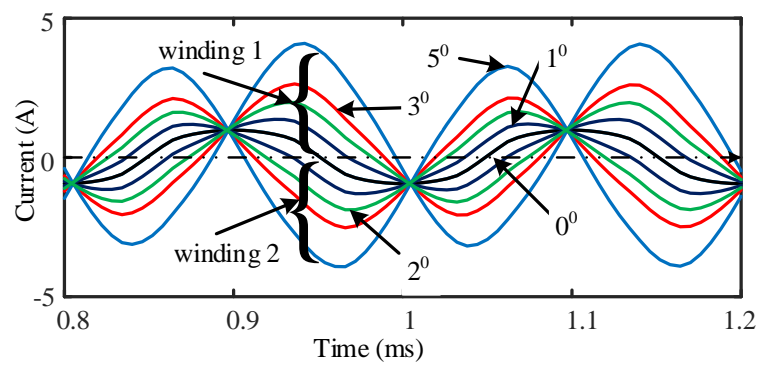

Fig. 4. The effect of phase change on load current.



Fig. 5. The magnetic flux density at $t=0.000852 \mathrm{~s}$ for $5^{\circ}$ phase difference between two sources.



Fig. 6 . The magnetic flux density at $t=0.000852 \mathrm{~s}$ for $0{ }^{\circ}$ phase difference between two sources.

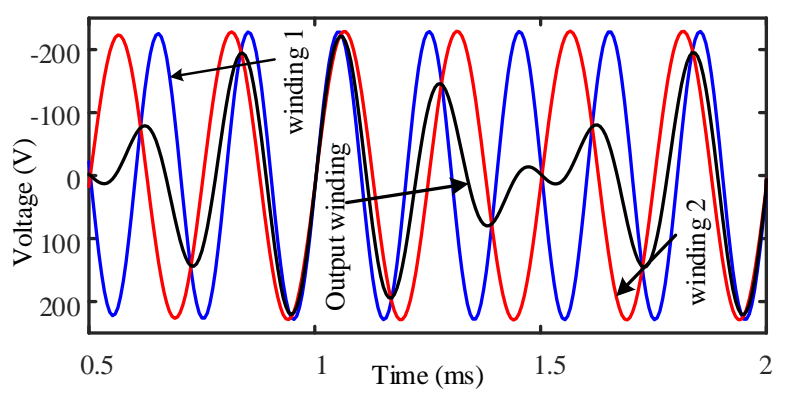

Fig. 7. The effect of frequency changes on induced voltage.
As the frequency difference between two input voltages are varied, the resultant induced voltage and current becomes distorted as shown in Fig. 7 and Fig. 8, respectively. Fig. 8 shows that a huge no-load currents flow between the windings, as one winding acts as a source and the other as a load. In the simulation, winding 1 and winding 2 are excited by $5 \mathrm{kHz}$ and $4 \mathrm{kHz}$, respectively, and winding 3 is open circuited. The frequency of the induced voltage becomes equal to the frequency difference between two sources. The corresponding magnetic flux density is shown in Fig. 9.

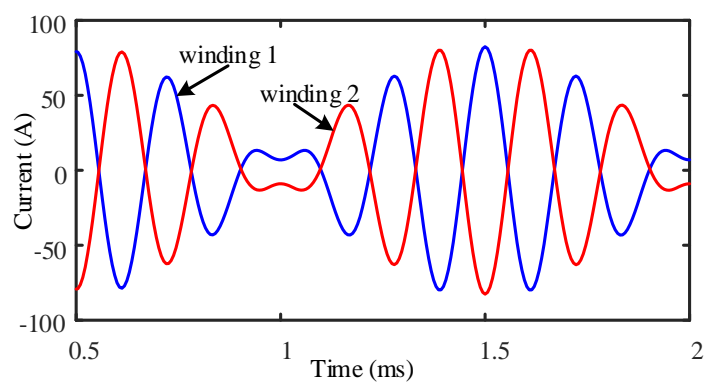

Fig. 8. The effect of frequency changes on the magnetizing currents.

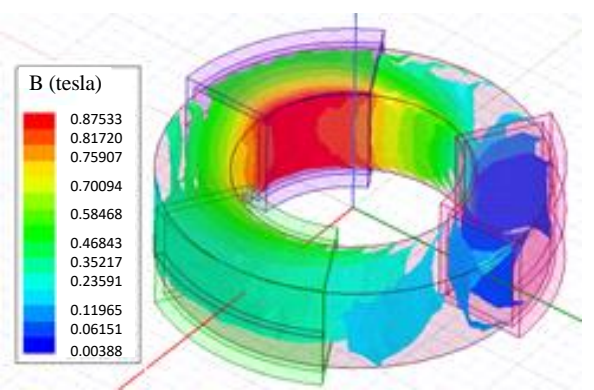

Fig. 9. The magnetic flux density at $t=0.000852 \mathrm{~s}$.

In the simulation, to observe the load sharing by the two sources, a $25 \Omega$ resistor is connected across the output winding. For the same voltage, phase and frequency, the two sources share equal load. To observe the effect of the voltage difference, winding 1 is excited by $230 \mathrm{~V}$ and winding 2 is excited by $200 \mathrm{~V}$. Fig. 10 shows that the winding with the higher voltage shares a greater load current than that of the lower voltage winding. A small phase shift exists between the excited windings, which indicates an extra current has been superimposed on the windings due to the changes in the magnitude of the winding 2 source voltage. The current in winding 2 , with the lower voltage excitation, increases due to the power exchanges among all windings, for the same load.

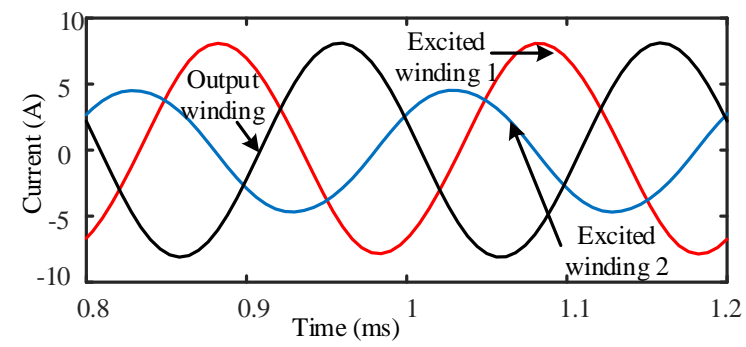

Fig. 10. The effect of higher voltage changes on the winding currents.

\section{EXPERIMENTAL INVESTIGATION AND DISCUSSIONS}

To validate the theoretical analysis, a prototype $2.5 \mathrm{kVA}$ 
high-frequency magnetic-link wound with nanocrystalline alloy is built in the laboratory. Fig. 11 shows the magneticlink with the nanocrystalline alloy-based core and the experimental test platform. Two windings were excited simultaneously with the California Instruments programmable power sources. The third winding is considered as the secondary winding and is used to measure the output. The Agilent Technologies DSO7034A oscilloscope with the KEYSIGHT N2791A differential voltage probes and the Agilent N2781A current probes are used to measure the voltage and currents.

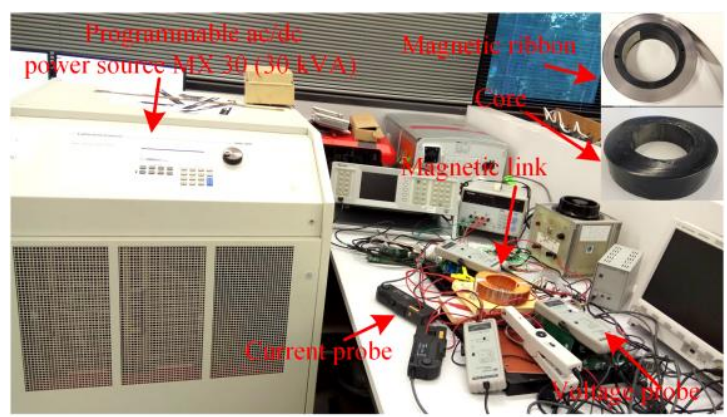

Fig. 11. Experimental test platform; programmable ac/dc power sources are used to excite the nanocrystalline magnetic-link.

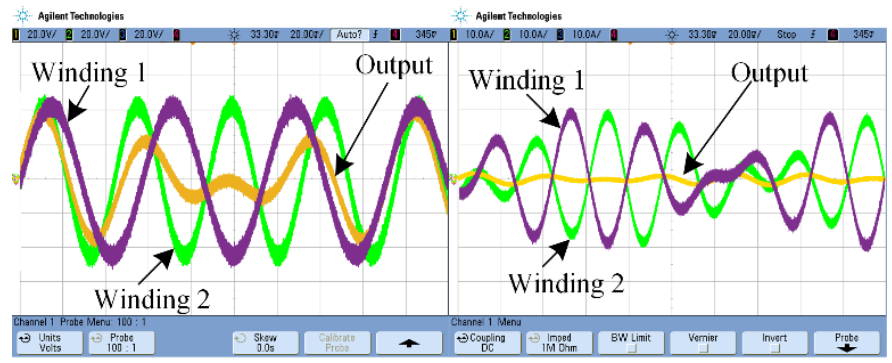

(a)

(b)

Fig. 12. Frequency difference effect between two windings: (a) voltage and (b) current



(a)

(b)

Fig. 13. Phase difference effect between two windings: (a) voltage and (b) current

Fig. 12(a) shows the effect of the phase difference between two input voltages on the output voltage. Windings 1 and 2 are excited with $50 \mathrm{~V}$ peak sinusoidal excitations of $800 \mathrm{~Hz}$ and $1000 \mathrm{~Hz}$ frequency, respectively. Fig. 12(a) shows that the output voltage peak varies significantly with time and gradually increases and decreases. It becomes approximately zero, when the two input voltages are totally opposite in phase. Fig. 12(b) shows the currents of the three windings. The frequency of the excitation voltage of winding 1 is kept lower than that of the winding 2 . The result is that the winding
1 current increases compared to that of winding 2 . The current in winding 1 increases as the frequency of the winding 2 voltage is increased further. The resultant output current diminishes when the two currents are in phase opposition. These experimental results in Figs. 12(a) and (b) validate the simulation results shown in Figs. 7 and 8. Fig. 13(a) shows the effect of the phase difference between the two excitations of winding 1 and 2 . In the experiment, the winding 2 voltage waveform leads the winding 1 voltage waveform. The output voltage is a constant sine wave and its peak will be dependent on the loading conditions. Fig. 13(b) shows the current performance under voltage excitation with difference phases. Fig. 13(b) shows that the lagging winding voltage draws more current than that of the leading one. The current difference between the two input windings can be increased by increasing the phase difference between the two voltages. The resultant current is a stable sinusoidal wave, which varies with the phase difference and the output load.

A good output voltage can only be obtained when both excitations are in phase and having the same frequency. A distorted voltage and a distorted current were observed when the excitation frequencies of the two voltages are not the same. The magnitude of the output voltage follows the source voltage with a higher excitation and decreases with the increase of the phase differences between the two exciting voltages and becomes lowest when they are out of phase.

\section{CONCLUSION}

This paper presents and discusses a systematic investigation of the magnetic response when multiple sources are interconnected through a common magnetic-link. The paper also presents the procedure used in the investigation. A mathematical model and a theoretical analysis of a multi-input high-frequency magnetic-link is presented. A simulation model has been developed in ANSYS environment for the systematic investigation of the magnetic response of a magnetic-link with multi-inputs. Different possible scenarios have been considered. Finally, the theoretical and simulation analysis have been validated with the experimental results of a nanocrystaline multi-input high-frequency magnetic-link built in the laboratory. It is observed that the effect of the voltage amplitude change is less than those from the frequency and phase angle change. Therefore, for unequal load sharing, the use of a small voltage change can be used to reduce the extra currents in the excited windings due to the unbalanced voltages. To connect the multiple sources by a magnetic link, several conditions need to be considered, such as the two voltages must have the same amplitude, the same frequency and the same phase angle. It is expected that the knowledge of the magnetic response, when multiple sources are interconnected with a common magnetic-link, will help the development of future green energy and smart grid technologies.

\section{REFERENCES}

[1] M. R. Islam, M. F. Rahman, and W. Xu, "Advances in Solar Photovoltaic Power Plants," Green Energy and Technology Series, Springer-Verlag GmbH, Heidelberg, Germany, Jun. 2016. 
[2] H. Dagdougui, R. Minciardi, A. Ouammi, M. Robba, and R. Sacile, "A dynamic decision model for the real-time control of hybrid renewable energy production systems," IEEE Systems J., vol. 4, no. 3, pp. 323-333, Sept. 2010.

[3] Y. Xu, N. Maki, and M. Izumi, "Performance comparison of 10-MW wind turbine generators with HTS, copper, and PM excitation," IEEE Trans. Appl. Supercond., vol. 25, no. 6, p. 5204006, Dec. 2015.

[4] J. S. Jeong, D. k. An, J. P. Hong, H. J. Kim, and Y. S. Jo, "Design of a 10-MW-class HTS homopolar generator for wind turbines," IEEE Trans. Appl. Supercond., vol. 27, no. 4, p. 5202804, Jun. 2017.

[5] Z. H. Chen, J. X. Jin, L. H. Zheng, and Z. H. Wu, "Advanced HTS dc transmission with self-protection function," IEEE Trans. Appl. Supercond., vol. 26, no. 7, p. 5403105, Oct. 2016.

[6] D. I. Doukas, A. Syrpas, and D. P. Labridis, "Multiterminal dc transmission systems based on superconducting cables feasibility study, modeling, and control," IEEE Trans. Appl. Supercond., vol. 28, no. 4, p. 5400506, Jun. 2018.

[7] J. X. Jin et al., "HTS power devices and systems: principles, characteristics, performance, and efficiency," IEEE Trans. Appl. Supercond., vol. 26, no. 7, p. 3800526, Oct. 2016.

[8] Z. Deng, J. Li, W. Zhang, Y. Gou, Y. Ren, and J. Zheng, "Hightemperature superconducting magnetic levitation vehicles: dynamic characteristics while running on a ring test line," IEEE Vehicular Tech. Magazine, vol. 12, no. 3, pp. 95-102, Sept. 2017.

[9] Z. Deng et al., "A high-temperature superconducting maglev-evacuated tube transport (HTS maglev-ETT) test system," IEEE Trans. Appl. Supercond., vol. 27, no. 6, p. 3602008, Sept. 2017.

[10] M. Jafari, Z. Malekjamshidi, G. Lei, T. Wang, G. Platt, and J. Zhu, "Design and implementation of an amorphous high-frequency transformer coupling multiple converters in a smart microgrid," IEEE Trans. Ind. Electron., vol. 64, no. 2, pp. 1028-1037, Feb. 2017.

[11] Y. Tang, J. Lu, B. Wu, S. Zou, W. Ding, and A. Khaligh, "An integrated dual-output isolated converter for plug-in electric vehicles," IEEE Trans. Vehicular Tech., vol. 67, no. 2, pp. 966-976, Feb. 2018.

[12] M. R. Islam, Y. G. Guo, and J. G. Zhu, "A multilevel medium-voltage inverter for step-up-transformer-less grid connection of photovoltaic power plants," IEEE J. Photovolt., vol. 4, no. 3, pp. 881-889, May 2014.
[13] R. W. A. A. De Doncker, D. M. Divan, and M. H. Kheraluwala, "A three-phase soft-switched high-power-density dc/dc converter for highpower applications," IEEE Trans. Ind. Appli., vol. 27, no. 1, pp. 63-73, Jan.-Feb. 1991.

[14] H. Tao, J. L. Duarte, and M. A. M. Hendrix, "Three-port triple-halfbridge bidirectional converter with zero-voltage switching," IEEE Trans. Power Electron., vol. 23, no. 2, pp. 782-792, Mar. 2008.

[15] L. F. Costa, G. Buticchi, and M. Liserre, "Quad-active-bridge dc-dc converter as cross-link for medium-voltage modular inverters," IEEE Trans. Ind. Appli., vol. 53, no. 2, pp. 1243-1253, Mar.-Apr. 2017.

[16] Y. Yu, G. Konstantinou, B. Hredzak, and V. G. Agelidis, "Power balance optimization of cascaded H-bridge multilevel converters for large-scale photovoltaic integration," IEEE Trans. Power Electron., vol. 31, no. 2, pp. 1108-1120, Feb. 2016.

[17] J. Rodriguez, S. Bernet, B. Wu, J. O. Pontt, and S. Kouro, "Multilevel voltage-source-converter topologies for industrial medium-voltage drives," IEEE Trans. Ind. Electron., vol. 54, no. 6, pp. 2930-2945, Dec. 2007.

[18] X. She, A. Q. Huang and R. Burgos, "Review of solid-state transformer technologies and their application in power distribution systems," IEEE J. Emer. Sele. Topics Power Electron., vol. 1, no. 3, pp. 186-198, Sept. 2013.

[19] M. R. Islam, Y. G. Guo, J. G. Zhu, H. Y. Lu, and J. X. Jin, "Highfrequency magnetic-link medium-voltage converter for superconducting generator-base high power density wind generation systems," IEEE Trans. Appli. Supercond., vol. 25, no. 5, p. 5202605, Oct. 2014.

[20] M. R. Islam, G. Lei, Y. G. Guo, and J. G. Zhu, "Optimal design of highfrequency magnetic-links for power converters used in grid connected renewable energy systems," IEEE Trans. Magn., vol. 50, no. 11, Nov. 2014.

[21] M. R. Islam, K. M. Muttaqi, D. Sutanto, and J. G. Zhu, "Design and implementation of amorphous magnetic material common magnetic bus for the replacement of common dc bus," IEEE Trans. Magn., vol. 54, no. 11, p. 2002004, Nov. 2018. 